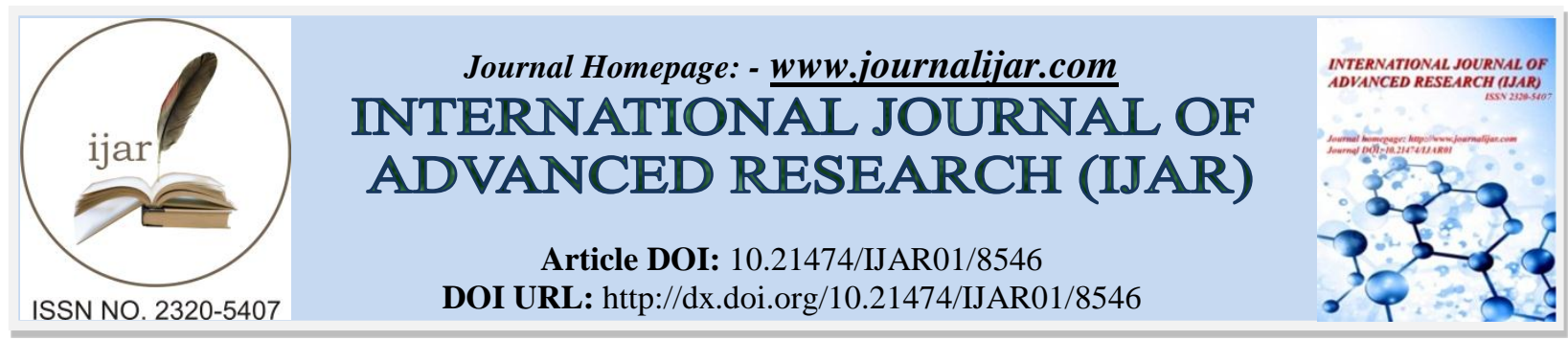

RESEARCH ARTICLE

\title{
CARDIOVASCULAR RHYTHMIC PACEMAKING ACTIVITIES OF SELECTED BOTANICAL EXTRACTS ON CARDIAC CHANNELOPATHY TACHYCARDIA ARRHYTHMIAS-INDUCED MYOGENICHEART USING IN VIVO MODEL OF FRESHWATER CRUSTACEAN DAPHNIA MAGNA (WATER FLEA).
}

Lorie E. Malaluan ${ }^{1}$ and Charmaine Aubrey G. Galindez ${ }^{2}$.

1. MIMAROPA Regional Science High School Pag-asa, Bansud, 5210 Oriental Mindoro Philippines.

2. University of Santo Tomas, España Blvd, Sampaloc, Manila, 1008 Metro Manila, Philippines.

\section{Manuscript Info}

…......................

Manuscript History

Received: 11 December 2018

Final Accepted: 13 January 2019

Published: February 2019

Key words:-

cardiovascular, arrhythmia, pacemaker, myogenic heart, heart diseases, antioxidant, minerals, secondary metabolites.

\begin{abstract}
Cardiac rhythmicity leads to sudden cardiac death which poses a unique challenge to lessen heart diseases that can cause sudden cardiac death in older and younger individuals. In vivo cardiovascular rhythmic pacemaking activities of selected botanical extracts on cardiac channelopathy tachycardia arrhythmias-induced myogenic heart was employed using in vivo model of freshwater crustacean daphnia magna (Water flea). Daphnia magna were cultured, maintained photoperiod at 16 hours light and 8 hours darkness before using as test specimens in the experimentation. Selected botanical extracts were used as treatment concentrations $(10 \%, 20 \%, 30 \%)$ for cardiotoxicity and cardio activity (heartbeats/min). A total of 250 test specimens or daphnids/neonates were collected and used in cardiotoxicity assessment of the selected botanical extracts after a $12 \mathrm{hr}$ time exposure to each treatment concentration solutions $(10 \%, 20 \%, 30 \%)$. Tachycardia arrhythmia was induced by a caffeine dilute solution concentration (30\%) in the bathing medium. Three hundred daphnids/neonates were used as test specimens in measuring the arrhythmias-induced myogenic heart of daphnids/neonates in ten replicates determination. Results showed that the selected botanicals are dose-dependent which means that the higher dosage the greater effect to the heartbeat rates. It was revealed that the losses of DPPH absorbance at $540 \mathrm{~nm}$ of the selected botanical extracts were in agreement with its anti-tachycardia arrhythmia effect to the heart rate of Daphnia magna. Results showed that the increase in treatment concentrations and time exposure had positive response against cardiac tachycardia arrhythmias-induced myogenic heart of Daphnia magna which behaves similarly to the heartrate of humans.
\end{abstract}

Copy Right, IJAR, 2019,. All rights reserved.

\section{Introduction:-}

The leading cause of death for all Americans and Filipinos is heart disease ${ }^{[1]}$. Cardiac events are the most common cause of death in persons with peripheral arterial disease ${ }^{[2]}$. It is classified that sudden cardiac arrest death is due to cardiomyopathies (mainly hypertrophic cardiomyopathy, dilated cardiomyopathy,and arrhythmogenic

Corresponding Author:-Lorie E. Malaluan.

Address:-MIMAROPA Regional Science High School Pag-asa, Bansud, 5210 Oriental Mindoro Philippines. 
cardiomyopathy) and channelopathies (mainly long QT syndrome, Brugada syndrome, short QT syndrome, and catecholaminergic polymorphic ventricular tachycardia). Cardiac channelopathies, are characterized by lethal arrhythmias in the structurally normal heart, incomplete penetrance, and variable expressivity. Cardiomyopathies, such as hypertrophic cardiomyopathy (HCM), dilated cardiomyopathy (DCM), and arrhythmogenic cardiomyopathy (AC) are characterized by the presence of structural heart alterations that lead to SCD-related arrhythmias ${ }^{[3]}$. Ventricular tachycardia and ventricular fibrillation are the two most frequent causes of sudden death. Chronic heart dysfunction can be caused by ventricular arrhythmias ${ }^{[4]}$.

There have been many researches that ion channel blockade has important limitations for chronic treatment and prevention of those arrhythmias. Limitations of current therapies for arrhythmia arise from the fact that these therapies do not address the underlying pathophysiology of arrhythmia. A more successful therapeutic approach may arise from targeting upstream pathologies that result in abnormalities in ionic currents and emergence of reentry and focal activity ${ }^{[5]}$. Cardiomyopathy, which is associated with significantly higher risk of VT/VF, is associated with oxidative stress and increased oxidation and carbonylation of proteins ${ }^{[6]}$.

In addition, other atrial fibrillation and heart failure, a condition with increased arrhythmic risk, are associated with excess amount of reactive oxygen free radicals (ROS). These mechanisms may involve the effect of ROS on genes, transcriptional regulation, protein trafficking, and posttranslational modifications ${ }^{[5]}$. Defibrillation has shown success in terminating VT/VF, nevertheless, it does not prevent the occurrence of arrhythmia, and frequent ICD shocks worsen the quality of life and may even increase mortality (Wissner et al., 2012) ${ }^{[5]}$. Thus, the molecular mechanism by which reactive oxygen free radicals (ROS) affect the cardiac Na+ current and how the Na+ current reduction can be prevented will be important steps toward designing new and effective antiarrhythmic drugs.

Numerous studies have been established that the pacemaking function of the heart is essential to normal cardiac activity. An incompetent heart in its contractile function leads to cardiac or ventricular arrest. If a pacemaking mechanism is absent, cardiac rhythmicity leads to sudden cardiac death which poses a unique challenge to lessen heart diseases that can cause sudden cardiac death in older and younger individuals. In addition, several researchers ${ }^{[7]}$ reported that there was a remarkable and alarming rate in sudden death of individuals within $24 \mathrm{~h}$ after being seen alive and in a normal state of health.

Interestingly, several studies have recognized that fruits are concentrated source of natural components thus having health promoting properties ${ }^{[8]}$. Malviya (2014) noted that fruits and vegetables are main source of natural antioxidant components. The antioxidants found in fruits and vegetables give protection against harmful free radicals and reduce rate of cancer and heart disease ${ }^{[9]}$. The most efficient carotenoid antioxidant is lycopene which can be mostly found in watermelon, tomato, papaya, and guava. Lycopene is a natural pigment which protects the body by neutralizing the negative effects of oxidants. It is a carotenoid and gives red color to vegetables and fruits. In the synthesis of vitamin A lycopene plays an important role as an intermediate and carotenoid like $\beta$-carotene and $\beta$ cryptoxent in, influences its development. Lycopene is soluble in fat and synthesized by plants and microorganisms ${ }^{[10]}$.

As with cardiac chanellopathies, phytochemical components of fruits rich in lycopene are of significant importance for various protective mechanisms through distinct pathways. Lycopene is a polyunsaturated hydrocarbon phytochemical present in red fruit and vegetables (papayas, tomatoes, red peppers, watermelons, etc.) and belongs to the tetraterpene carotenoid family ${ }^{[11]}$. Moreover, lycopene provides mechanism in cancer prevention, reduces LDL cholesterol and cardiovascular diseases.

There have been many researchers who are focusing on exploitation of natural resources for dietary regimen against life threatening ailments ${ }^{[12,13]}$. Accumulating research evidence suggests that intake of lycopene from tomatoes and tomato-based products reported associations between tomato intake and risk of cardiovascular diseases ${ }^{[14]}$. The lycopene biosynthesis of fruits is found predominantly during the ripening process, thus increasing the lycopene content. These functional ingredients act as protection against chronic health problems like cancer insurgence and cardiovascular disorders $\left.{ }^{[15,16]}\right]$. The low toxicity and high tolerance of lycopene open the door to various options in the design of lycopene supplementation protocols.

In addition, a recent study has gained interest by investigating the effect of mangiferin on the isoproterenol- induced myocardial infarction in rats. Mangiferin was found to ameliorate the effect of isoproterenol-induced pathological 
changes, reduced the lipid peroxide formation and retained the myocardial marker enzyme activities at near normal level. Mangiferin has been considered as a candidate for immunoregulators which is good for the heart ${ }^{[17]}$.

Although very limited and preliminary, the natural compound, mangiferin, has interesting potential in treatments of metabolic syndromes via modulation of multiple biological processes. It is the predominant constituent of Mangifera indica extract from the plant that produces mango. Mango extracts and the mango component mangiferin promote endothelial cell migration ${ }^{[18]}$. In this present study, Mangifera indica leaves had been used since mangiferin content is in the range of $0.3 \%-1 \%(\mathrm{w} / \mathrm{w})$ in peel and less than $1 \%$ in flesh ${ }^{[19]}$. Leaves of Mangifera indica $\mathrm{L}$. are a rich source of phenolic compounds. Mangiferin obtained from the leaves and bark of the tree, is a xanthone with wide range of pharmacological effects ${ }^{[20]}$. Moreover, mangiferin is a highly potent antioxidant present in mango leaves which is utilized for therapeutic purposes ${ }^{[21]}$.

Apparently, invasive studies and evaluations of recent researches are frequently employed using animal studies ${ }^{[22]}$. Since, the myogenic heart of the water flea Daphnia magna is regulated by inhibitory cholinergic neurons that modulate cardiac function, including heart rate, Daphnia magna is a useful model organism for cardiovascular function, due to its physical transparency and susceptibility to cardioactive drugs known to affect the human heart

${ }^{[23]}$. Unlike neurogenic hearts, in which the cardiac ganglion acts as the pacemaker to initiate contraction, myogenic heart rhythms are initiated in the cardiac muscular tissue and are independent of neural, metabolic, or hormonal stimulation ${ }^{[24]}$. Their physical transparency allows for simple observation of internal structures, including the heart ${ }^{[25]}$. In addition, they are highly sensitive to environmental changes, which cause them to adapt morphologically, physiologically, and behaviorally ${ }^{[26]}$.

The heart of the Daphnia magna, regulated by cholinergic neurons and may be useful as a model for the effect of drugs on cardiovascular function and unusual among crustaceans in that they possess myogenic hearts. Its heart is composed of a thin layer of myocardial cells, making it easily modulated by small concentrations of cardioactive compounds ${ }^{[27]}$.Testing the effects of the drugs is simplified in Daphnia magna as the fleas are responsive to pharmacological agents added to the water in which they swim. Thus, the introduction of these pharmacological agents to water fleas may induce activity directly on the cardiac muscle ${ }^{[28]}$.

Numerous investigations have been made on lycopene and mangiferin and its potential for therapeutic benefits and reduced cardiovascular diseases. However, the underlying mechanisms of lycopene and mangiferin to cardiac chanellopathies are not clearly understood which motivated the present study. Very little is known about the potential mechanism of its effects on cardiac channelopathies using animal models to investigate in vivo biochemical functions.

In the present study, the cardiac channelopathies effects of ethanolic extracts of fruit pulp of tomato and papaya, and the ethanolic extract of mango leaves on Daphnia magna were tested. Extracted samples mentioned earlier have not been tested in any in vivo animal model prior to this study. Thus, in vivo cardiovascular rhythmic pacemaking activities of selected botanical extracts had been investigated against cardiac channelopathy tachycardia arrhythmias-induced myogenic heart using freshwater Crustacean Daphnia magna (Water flea). Daphnia magna has myogenic heart tissue that is similar to mammals ${ }^{[29]}$. Thus, it was necessary first to clarify the cardiac channelopathies mechanisms of the Daphnia magna heart, which has a myogenic heart with a cardiac pacemaker similar to the hearts of vertebrates ${ }^{[30]}$ to establish its worth as a useful model organism.

The cardiovascular rhythmic pacemaking activities of Solanum lycopersicum (Tomato) and Carica papaya (Papaya), and Mangifera indica L. (Mango) leaves on the heart rate of Daphnia magna and its implication on cardiotoxicity were assessed to provide a better understanding of the sympathetic pacemaking mechanism regulation of the Daphnia magna heart which behaves similarly to the heart rate of humans.

\section{Materials and Methods:-}

\section{Fruit Harvesting, Leaf Collection and Verification}

Fruits of tomato Solanum lycopersicum (tomato), Carica papaya (papaya), and leaves of Mangifera indica $L$. (mango) were harvested in acceptable proportions at Roxas, Bongabong, and Bansud, Oriental Mindoro. Samples were sent to the Bureau of Plant Industry in Manila for correct identification and verification. 
Culture of Daphnia magna (Water flea) and its Verification

Daphnia magna (Water flea) had been obtained from an aquaculturist from Dumaguete City, Negros Oriental. Freshwater Crustacean Daphnia magna (Water flea) species were identified and verified by an aquaculturist from Tilapia Hatchery under the Bureau of Fisheries and Aquatic Resources located at Barcenaga, Naujan, Oriental Mindoro. Daphnia magna specimens were cultured in a rectangular plastic container just to keep better track visually on the culture's progress and estimating how much to feed. Daphnia magna were maintained photoperiod at 16 hours light and 8 hours darkness. Culturing of Daphnia magna was located at a cool location with water temperature between $65^{\circ} \mathrm{C}$ and $70^{\circ} \mathrm{C}$ which is ideal for Daphnia magna reproduction. Yeast was used as feeds for Daphnia magna. Feeding, water change and general culture health parameters are recorded on data sheets and was stored/archived for reference.

Preparation of Ethanolic Extraction of Fruit Pulp Extract of Solanum lycopersicum (Tomato) and Carica papaya (Papaya) and Mangifera indica L. (Mango) Leaves

The collected Mangifera indica L. (Mango) leaves were washed in water, shade dried for one week at room temperature. Mangifera indica L. (Mango) leaves were macerated in ethanol for at least 48 hours in readiness for rotary evaporation. Meanwhile, fresh fruit pulp was taken from ripe Solanum lycopersicum (Tomato) and Carica papaya (Papaya), macerated in ethanol for at least 48 hours and was then filtered using Whatman filter paper no.1. The ethanolic solution was allowed to evaporate using water bath at $50^{\circ} \mathrm{C}$ until dried extract/crude extract was obtained.

\section{Preliminary Phytochemical Screening}

Preliminary phytochemical screening of the lycopene extracted from Solanum lycopersicum (Tomato) and Carica papaya (Papaya) and the Mangifera indica L. (Mango) leaf ethanolic extract was carried out to find out the presence of various secondary metabolites (tannins, alkaloids, saponins, flavonoids, steroids, terpenoids, anthraquinones, reducing sugars and glycosides) using standard procedures ${ }^{[31,32,33]}$. The qualitative results are expressed as (+) for the presence and $(-)$ for the absence of phytochemicals.

Cardiotoxicity assessment of Ethanolic Crude Extract of Solanum lycopersicum (Tomato), Carica papaya (Papaya), and the Ethanolic Extract of Mangifera indica L. (Mango) Leaves

Daphnia magna underwent a $12 \mathrm{hr}$ time exposure at various treatment solution concentrations $(100,200,300 \mathrm{mg} / \mathrm{L})$. Daphnia magna neonates (one day old daphnids) had been selected for this assessment since neonates may be more susceptible to toxic substance than elder one ${ }^{[34]}$. No food feed was provided during the experimental period. Death and immobilization of daphnids at a $12 \mathrm{hr}$ time exposure was thoroughly observed in a digital microscope connected to a laptop. No food feed was administered during the cardiotoxicity assessment of Daphnia magna (water flea). Different treatment solution concentration $(10 \%, 20 \%, 30 \%)$ of at least $2 \mathrm{~mL}$ test solution should be provided for each Daphnia magna was added directly to the petri dish as test chambers at various concentrations. Five daphnids/neonates were put into each test chamber per concentration and were used in cardiotoxicity assessment in five replicates determination. Thus, a total of 250 test specimens or daphnids/neonates were collected and used to determine the mortality rate after a $12 \mathrm{hr}$ time exposure to each treatment concentration solutions $(10 \%, 20 \%, 30 \%)$.

In Vivo Cardio Activity Assessment on the Test specimens Daphnia magna (Water flea)

Arrhythmia was induced by a caffeine dilute solution in the bathing medium. Three caffeine solutions were created at three various dilute solution concentrations $(10,20,30 \mathrm{mg} / \mathrm{mL})$. Three daphnids/neonates were used as test specimens in the observation of heart beat per minute per dilute solution concentration. Examination of daphnids/neonates were observed a 10 minute time exposure since these test specimens showed an increase response in their heart beat per minute in the $10 \mathrm{mg} / \mathrm{mL}$ caffeine dilute solution. Hence, a $10 \mathrm{mg} / \mathrm{mL}$ caffeine dilute solution was made as a standard bathing medium in increasing the heart beat per minute of the test specimens daphnids/neonates used in this current study. All experimental or control groups had been exposed to the bathing medium of $10 \mathrm{mg} / \mathrm{mL}$ caffeine dilute solution. Daphnia magna was carefully picked up by a plastic pipette from a container and transferred it individually into the bathing medium. The picking up of the test specimen was carried out cautiously with minimal volume of liquid sucked in the pipette. Individual Daphnia magna was exposed into the bathing medium for 10 minutes. Daphnia magna in the control condition were kept in distilled water while experimental condition Daphnia magna was administered with appropriate treatment solutionconcentration (10\%, $20 \%, 30 \%)$. 
Daphnia magna from the culture water provided this present study with a value for the normal resting heart rate of Daphnia magna. Daphnia magna was transferred individually by a pipette to the petri dish as test chamber. Due to lack of previously reported information on its lethal dose in Daphnia magna, a starting concentration of $100 \mathrm{mg} / \mathrm{L}$ of treatment solution concentration was chosen so that the highest concentration did not $300 \mathrm{mg} / \mathrm{L}$. Test specimens were tested at concentrations not exceeding $1 \mathrm{~g} / \mathrm{L}$ since daphnids/neonates sizes are 2-3 mm only. Different treatment solutions of $100 \mathrm{mg} / \mathrm{L}, 200 \mathrm{mg} / \mathrm{L}, 300 \mathrm{mg} / \mathrm{L}$ with different time exposure ( $3 \mathrm{~min}, 5 \mathrm{~min}, 10 \mathrm{~min}$ ) resulted in 100, 200, and 300 microgram per $\mathrm{mL}$ of concentrations available to the Daphnia magna in each exposure. At least $2 \mathrm{~mL}$ of treatment solution concentration was provided for each Daphnia magna specimen.

Three hundred petri dishes and 300 daphnids/neonates were used as test chambers and test specimens in this current study in measuring the arrhythmias-induced myogenic heart of daphnids/neonates. Each treatment solution concentration was done in ten replications.

The heart rates of Daphnia magna were observed using a digital/video microscope connected to a laptop. To control the movement of the test specimen, the test specimen Daphnia magna was placed on a depression slide coated with petroleum jelly. Each test specimen was transferred to the depression slide by using a plastic pipette. Heart rate was measured ten times for each time exposure with regards to its respective treatment solution concentration.

Trials were completed with the room lights off and only the light from the digital/video microscope were on because it can cause stress to the Daphnia magna which are sensitive to light contact. A consistent and systematic way of counting method was employed in this current study in counting the fast heartbeats of Daphnia magna by videotaping the heart beat and slowing the recording to obtain a more precise count.

\section{DPPH Antioxidant Assay and Inhibitory Activities of Selected Botanical Extracts}

The method was adapted from Clarke et al. (2013), twenty microliter $(20 \mu \mathrm{L})$ of the crude extract diluted appropriately in Dimethylsulfoxide (DMSO) with $180 \mu \mathrm{L}$ of DPPH in methanol $(40 \mu \mathrm{g} / \mathrm{mL})$ in wells of a $96-w e l l$ plate. The plate was kept in the dark for 15 minutes after which the absorbance of the solution was measured at 540 nm in a plate reader. DMSO served as a blank and Ascorbic Acid served as the standard.

$$
\% \text { inhibition }=\frac{\text { Absorbance of DPPH }- \text { Absorbance of extact }}{\text { Absorbance of DPPH }} \times 100
$$

\section{H. Elemental Analysis by XRF Spectrometer}

Trace elements were quantitatively determined in the crude ethanolic fruit extract of Solanum lycopersicum (tomato), Carica papaya (papaya) and Mangifera indica L. (mango) ethanolic leaf extract by X-Ray flouresence spectrometer (XRF).

\section{Data Analysis}

Mean averages were used in comparing results of cardiotoxicity and the heartbeats/per minute were obtained from the selected botanical extracts with different treatment concentrations. Meanwhile, One-Factor Analysis of Variance (ANOVA) was applied in determining the possible differences obtained from the results of the experimentations.

\section{Results and Discussion:-}

Table 1:- Qualitative phytochemical analysis of the crude ethanolic fruit extract of Solanum lycopersicum (tomato), Carica papaya (papaya) and Mangifera indica L. (Mango) ethanolic leaf extract

\begin{tabular}{|c|c|c|c|}
\hline Phytochemicals & Tomato & Papaya & Mango \\
\hline Tannins & + & + & + \\
\hline Carotenoid & + & + & - \\
\hline Alkaloid & + & - & + \\
\hline Cardiac glycoside & + & - & + \\
\hline Terpenoids & + & + & + \\
\hline Saponins & + & + & + \\
\hline Flavonoids & + & + & + \\
\hline Steroids & - & + & - \\
\hline
\end{tabular}




\begin{tabular}{|c|c|c|c|}
\hline Reducing sugar & - & - & + \\
\hline Anthraquinones & - & - & + \\
\hline Phenol & - & - & + \\
\hline Xanthoprotein & - & - & + \\
\hline
\end{tabular}

Key: (+) Presence, (-) Absence

The qualitative phytochemical screening analysis of the crude ethanolic fruit extract of Solanum lycopersicum (tomato), Carica papaya (papaya) and Mangifera indica L. (Mango) ethanolic leaf extract showed the presence of tannins, cardiac glycoside, saponins, and flavonoids (Table 1), however, the presence of carotenoid has only been found in Solanum lycopersicum (Tomato) and Carica papaya (Papaya). Result in Table 1 also indicated the presence of alkaloids and terpenoids in both Solanum lycopersicum (Tomato) and Mangifera indica L. (Mango) ethanolic leaf extract. On the other hand, Carica papaya (Papaya) and Mangifera indica L. (Mango), showed the occurrence of secondary metabolite steroids. Preliminary screening also showed a manifestation of carbohydrates in Carica papaya (Papaya) only, whereas, the existence of reducing sugars, anthraquinones, and xanthoproteins were found to be in Mangifera indica L. (Mango) ethanolic leaf extract that were not detected in the crude ethanolic fruit pulp extract of Solanum lycopersicum (Tomato) and Carica papaya (Papaya).

Table 2:- $12 \mathrm{hr}$ Cardiotoxicity Assessment in different concentration of the crude ethanolic fruit extract of Solanum lycopersicum (Tomato), Carica papaya (Papaya) and Mangifera indica L. (Mango) Ethanolic Leaf Extract on the Freshwater Crustacean Daphnia magna (Water flea)

\begin{tabular}{|c|c|c|c|c|c|c|c|c|c|c|}
\hline & \multirow[t]{2}{*}{ Control } & \multicolumn{3}{|c|}{$\begin{array}{c}\text { Solanum lypersicum } \\
\text { (Tomato) }\end{array}$} & \multicolumn{3}{|c|}{ Carica papaya (Papaya) } & \multicolumn{3}{|c|}{$\begin{array}{c}\text { Mangifera indica } L . \\
\text { (Mango) }\end{array}$} \\
\hline & & \multicolumn{9}{|c|}{ Concentration $(\mathrm{mg} / \mathrm{L})$} \\
\hline Replication & & $10 \%$ & $20 \%$ & $30 \%$ & $10 \%$ & $20 \%$ & $30 \%$ & $10 \%$ & $20 \%$ & $30 \%$ \\
\hline 1 & 5 & 5 & 5 & 5 & 5 & 5 & 5 & 5 & 5 & 5 \\
\hline 2 & 5 & 5 & 5 & 5 & 5 & 5 & 5 & 5 & 5 & 5 \\
\hline 3 & 5 & 5 & 5 & 5 & 5 & 5 & 5 & 5 & 5 & 5 \\
\hline 4 & 5 & 5 & 5 & 5 & 5 & 5 & 5 & 5 & 5 & 5 \\
\hline 5 & 5 & 5 & 5 & 5 & 5 & 5 & 5 & 5 & 5 & 5 \\
\hline Mean & 5 & 5 & 5 & 5 & 5 & 5 & 5 & 5 & 5 & 5 \\
\hline $\begin{array}{c}\text { Corrected \% } \\
\text { Mortality }\end{array}$ & $0 \%$ & $0 \%$ & $0 \%$ & $0 \%$ & $0 \%$ & $0 \%$ & $0 \%$ & $0 \%$ & $0 \%$ & $0 \%$ \\
\hline
\end{tabular}

Overall, it was indicated in Table 2 that there was a corrected $0 \%$ mortality which means that not even one Daphnia magna died during the 12 hour time exposure in different treatment concentrations $(10 \%, 20 \%, 30 \%)$ of the crude ethanolic fruit extract of Solanum lypersicum (Tomato), Carica papaya (Papaya) and Mangifera indica L. (Mango) ethanolic leaf extract in acute toxicity assessment. Acute toxicity assessment performed reveals that it is non-toxic in nature and safe for freshwater crustacean Daphnia magna (Water flea). Since Daphnia magna (water flea) has similar myogenic heart as compared to human heart, it can be inferred from the result of this current study that the crude ethanolic fruit extract of Solanum lycopersicum (Tomato), Carica papaya (Papaya) and Mangifera indica L. (Mango) ethanolic leaf extract manifest cardio protective effect without any toxicity. Species of crustaceans Daphnia magna shows high tolerance on different concentrations of selected plant extracts (tomato, papaya, mango) during the $12 \mathrm{hr}$ exposure. Results of mortality rate remain unchanged as the concentrations of plant extracts increases during the 12-hr time exposure of Daphna magna which stipulated a zero corrected \% mortality using Abbot's formula and confirmed its potential as a safe pharmacological agent. 

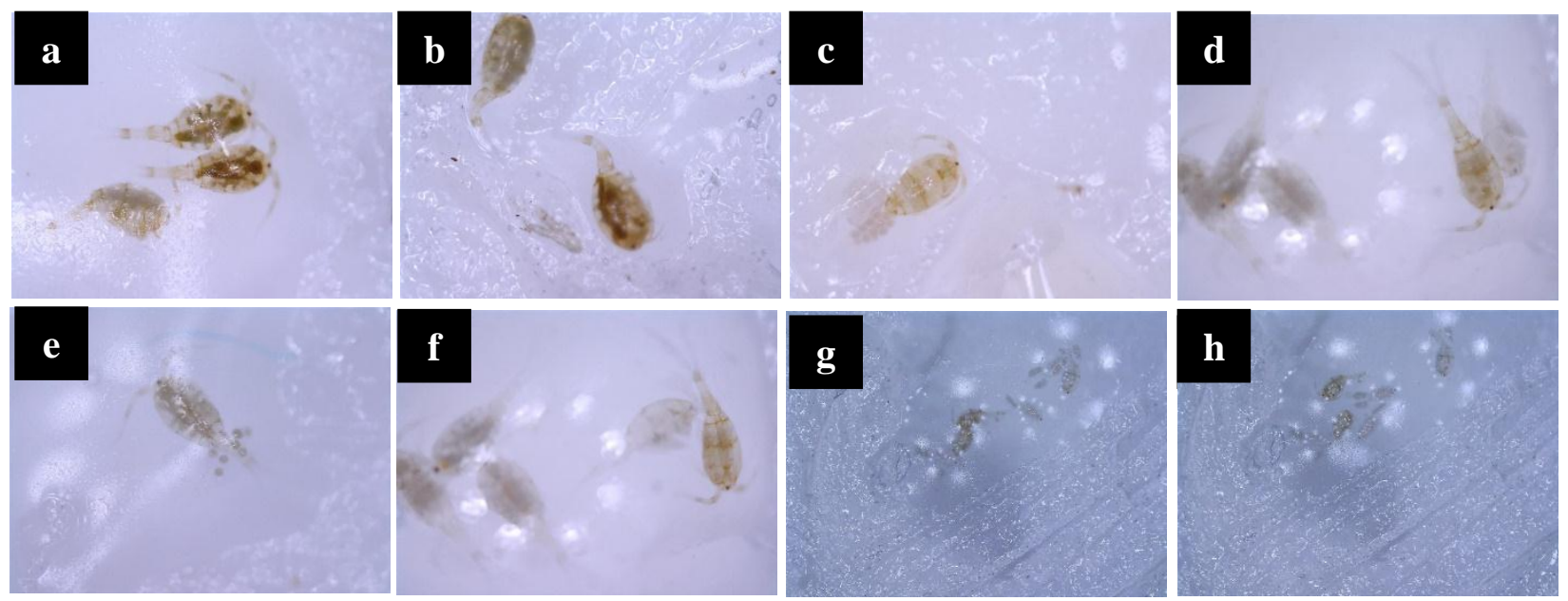

Figure 1:- Freshwater Daphnia magna (water flea) neonates or daphnids picked up from the petri dish during cardiotoxicity assessment at 500X Magnification (a, b, c, d, e, f) and 200x magnification ( $\mathrm{g}, \mathrm{h})$

Heart rate of Daphnia magna under control and different concentration of the crude ethanolic fruit extract of Solanum lycopersicum (Tomato), Carica papaya (Papaya) and Mangifera indica L. (Mango) Ethanolic Leaf Extract in different time exposure ( $3 \mathrm{~min}, 5 \mathrm{~min}, 10 \mathrm{~min}$ )

One-Factor ANOVA results show significant difference among the different concentrations of selected botanical extracts in terms of heartbeat rate within 3-minute $(\mathrm{F}=108.818 ; \mathrm{p}=0.000), 5$-minute $(\mathrm{F}=310.713 ; \mathrm{p}=0.000)$, and 10minute time of exposure $(\mathrm{F}=1227.212 ; \mathrm{p}=0.000)$ wherein the $30 \%$ of Mangifera indica L. (Mango) extract exhibited the lowest average heartbeat rates $(M=171.4 ; M=144 ; M=124)$ while highest average heartbeat rate was demonstrated by the control $(\mathrm{M}=189.4 ; \mathrm{M}=189.9 ; \mathrm{M}=188.4)$ for all the exposure time applied. The results indicated that the selected botanicals are dose-dependent which means that the higher dosage, the greater effect to the heartbeat rates.

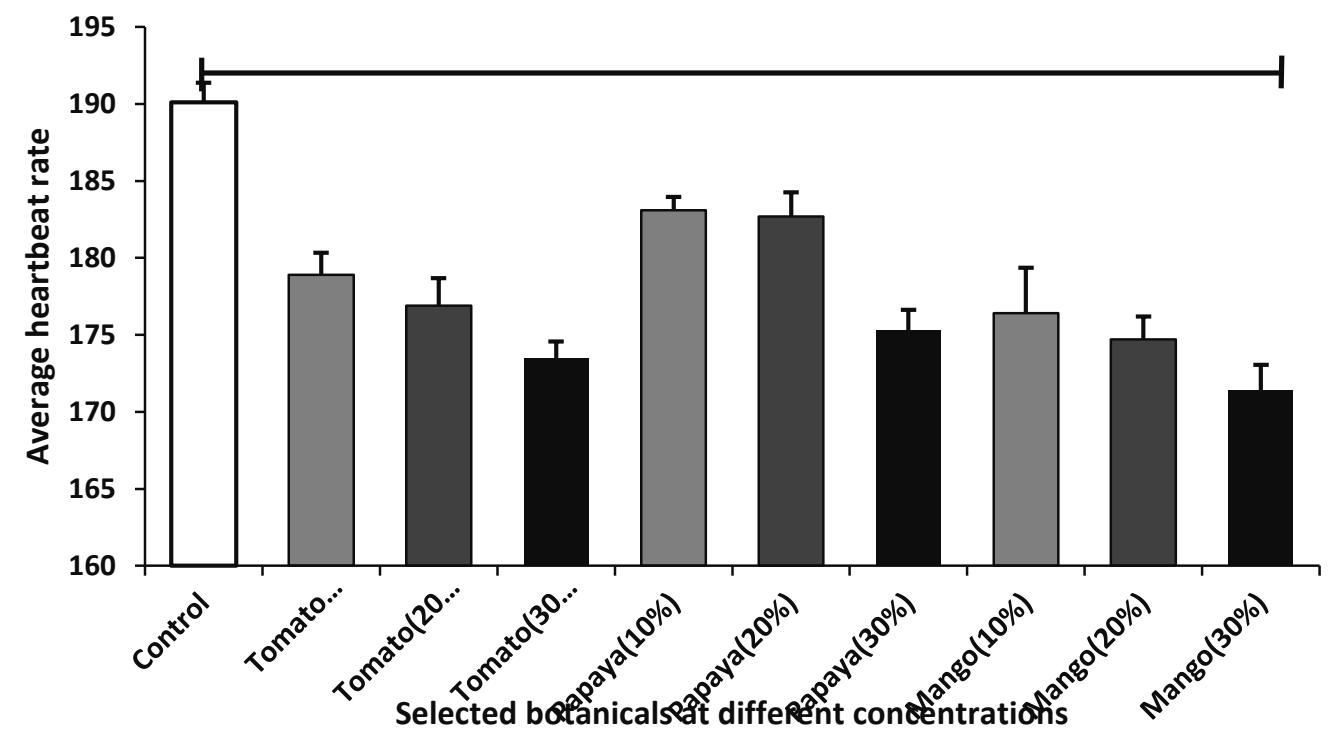

Figure 2:- Heart rate of control and different treatment concentration of T1 (Solanum lypersicum), T2 (Carica papaya) and T3 (Mangifera indica L.) on the myogenic heart of freshwater crustacean Daphnia magna (Water flea) during the 3-minute time exposure period 
Average heart rates for the control (190.1 heartbeats per minute) were higher than all of the other treatments (T1, $\mathrm{T} 2$, T3). The mean heartbeat of control, T1 of $10 \%, 20 \%, 30 \% \mathrm{mg} / \mathrm{L}$ concentrations at 3 min time exposure were found to be 178.9, 176.9 and 173.5 respectively. Meanwhile, T2 of 10\%, 20\%, 30\% depicts mean heartbeats of 183.1, 182.7, and 175.3. T3 of 10\%, 20\%, 30\% show mean heartbeats of 176.4, 174.7 and 171.4 respectively.

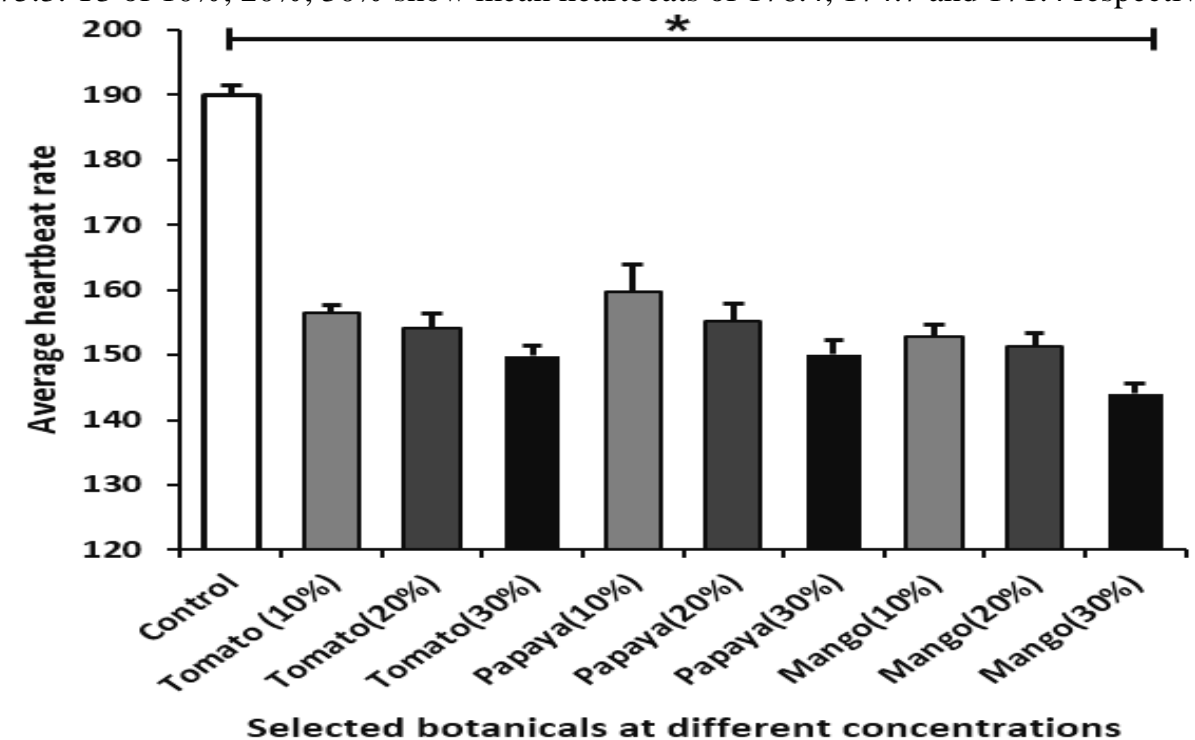

Figure 3:- Heart rate of control and different treatment concentration of T1 (Solanum lycopersicum), T2 (Carica papaya) and T3 (Mangifera indica L.) on the myogenic heart of freshwater crustacean Daphnia magna (Water flea) during the 5-minute time exposure period

Similarly, the average heart rates for the control (189.9 beats per minute) were higher than all of the other treatments (T1, T2, T3). Figure 4 displays mean heartbeats obtained at various concentrations exposed to a 5 minute time exposure period. A mean heartbeat per minute of 156.4, 154.1, and 149.9 were obtained by Daphnia magna exposed to $\mathrm{T} 1(10 \%, 20 \%, 30 \%)$. Meanwhile, it was depicted that a mean value heartbeats per minute of 159.7, 155.1, and 150.1 was observed on the Daphnia magna present in the test chambers of T2 (10\%, 20\%, 30\%). Moreover, T3 $(10 \%, 20 \%, 30 \%)$ shows apparent mean heartbeats per minute of 152.8, 151.4, and 144.0.

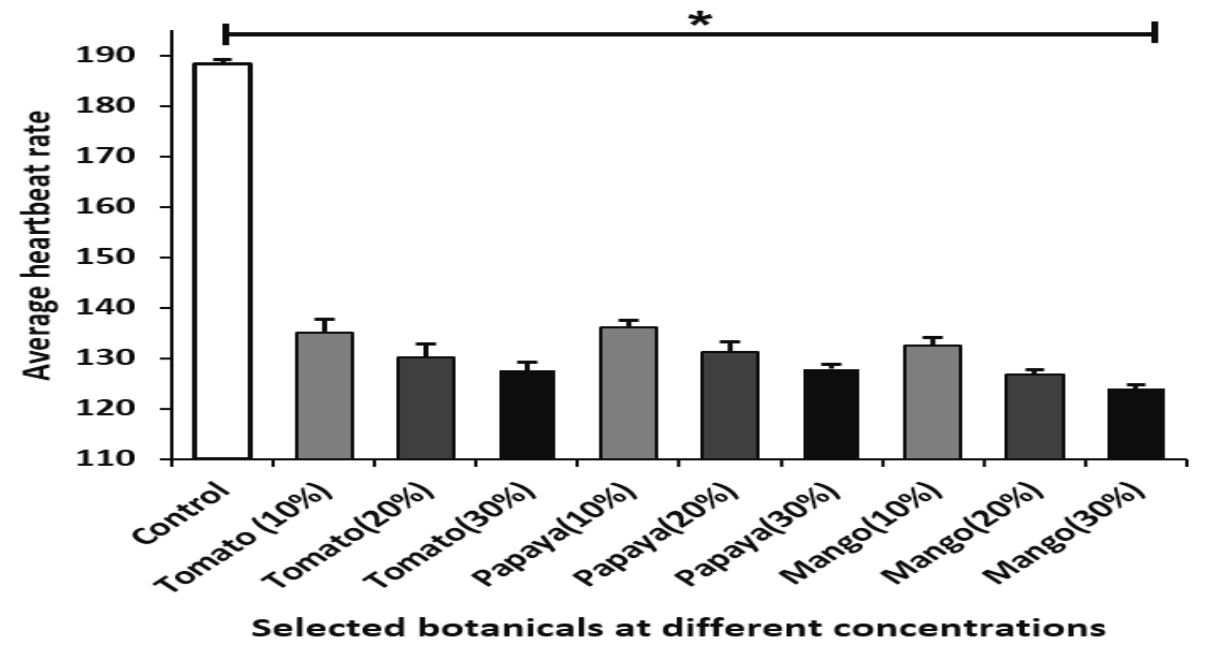

Figure 4:- Heart rate of control and different treatment concentration of T1 (Solanum lycopersicum), T2 (Carica papaya) and T3 (Mangifera indica L.) on the myogenic heart of freshwater crustacean Daphnia magna (Water flea) during the 10-minute time exposure period

Similarly, the average heart rates for the control (188.4 heartbeats per minute) were higher than all of the other treatments (T1,T2, T3). There were minimal changes in the average heart rate of the test specimen in the control group. Mean heartbeat per minute of Daphnia magna exposed at various concentrations at 10 minute time exposure 
was shown in Figure 5. Mean heartbeat values of 135.1, 130.2, and 127.6 per minute were observed under T1 (10\%, $20 \%, 30 \%)$. On the other hand, for T2 (10\%, 20\%, 30\%), the mean values of the heartbeat of Daphnia magna were 136.1, 131.2, and 127.9 per minute. Meanwhile, it was also observed that mean heartbeat values of Daphnia magna for T3 $(10 \%, 20 \%, 30 \%)$ were $132.67,126.7$, and 124.0 per minute respectively.

Nevertheless, it was shown in figures 3, 4, and 5 the heartbeats/ minute of the myogenic heart of Daphnia magna induced by cardiac channelopathy arrhythmias heart in response to different concentrations of the crude ethanolic fruit extract of Solanum lycopersicum (Tomato), Carica papaya (Papaya) and Mangifera indica L. (Mango) Ethanolic Leaf Extract at various time exposure period on the ( $3 \mathrm{~min}, 5 \mathrm{~min}, 10 \mathrm{~min})$. The results clearly showed the dose dependent cardioprotective effect of the selected different plant extracts on Daphnia magna (Figure 3, 4, 5). In the case of the crude ethanolic extract of Solanum lypersicum (Tomato), Carica papaya (Papaya) and Mangifera indica L. (Mango), it was clearly manifested that the heart beats of Daphnia magna decreases as the concentration of plant extract increases with regards to its exposure time period. The Mangifera indica L. (Mango) leaves crude ethanolic extract showed the most considerable decrease of heart beats of Daphnia magna among the selected plant extracts used in this present study.

Nevertheless, the decrease of heart rate per minute of the test specimens are associated to the phytochemicals present in the selected plant extracts of Solanum lycopersicum (Tomato), Carica papaya (Papaya) and Mangifera indica L. (Mango). One of the phytochemicals present in all plant extracts used in this current study shows the presence of cardiac glycosides. Several studies have established that frequently used classes of drugs for the treatment of heart failure and arrhythmias have been taken from plants rich in phytochemicals. Cardiac glycosides work by inhibiting the $\mathrm{Na}+\mathrm{K}+$ pump. This causes an increase in the level of sodium ions in the myocytes, which then leads to a rise in the level of calcium ions. This inhibition increases the amount of Ca2+ ions available for contraction of the heart muscle, improves cardiac output and reduces distention of the heart ${ }^{[35]}$. Thus, cardiac glycoside counteract atrial fibrillation by slowing down heart rate which aid in the treatment of congestive heart failure and cardiac arrhythmias, which was parallel to the result of this current study.

Moreover, results of the decrease heartbeats can also be attributed to the interesting pharmacological effects including negative chronotropic, diuretic, antibacterial and anti-inflammatory actions associated with phytochemical saponins ${ }^{[36]}$. The non-sugar part of saponins also has antioxidant activity which may help to reduce risk of heart diseases ${ }^{[37,38]}$. In addition, certain active secondary metabolites such as saponins, alkaloids, phenolic compounds, cardiac glycosides and flavonoids are recently gaining more attention for treating patients with cardiovascular diseases heart disease ${ }^{[39]}$.

Similarly, flavonoids and phenolic compounds have also been reported to produce a decrease in heart rate and vasodilation in animal models ${ }^{[40,41]}$. These compounds have been studied mainly for their properties against oxidative damage leading to various degenerative diseases, such as cardiovascular diseases, inflammation and cancer ${ }^{[42]}$. Moreover, flavonoids contain free radical damage is believed to contribute to a variety of health problems, including cancer, heart disease and aging ${ }^{[43]}$. Study findings suggest that phytochemicals may reduce the risk of coronary heart disease by preventing the oxidation of low density lipoprotein (LDL) cholesterol, reducing the synthesis or absorption of cholesterol, normalizing blood pressure and clotting, and improving arterial elasticity ${ }^{[44,}$ ${ }^{42]}$. Flavonoids prevent the oxidation of low-density lipoprotein, lowers the blood levels of cholesterol and triglycerides thereby reducing the risk for the development of atherosclerosis ${ }^{[45]}$. Flavonoids have also been reported to have vaso-dilatory and inhibitory effects on platelet aggregation thereby preventing coronary heart diseases ${ }^{[46]}$.

On the other hand, the response of the test specimens on treatments (T1, T2, T3) could be attributed to the presence of phenolic compound found T1 and T3. In the case of T3, leaves of Mangifera indica L. (Mango) are rich source of phenolic compounds ${ }^{[19]}$. Likewise, mangiferin obtained from the leaves is a xanthone with wide range of pharmacological effects ${ }^{[20]}$. This active pytochemical is a highly potent antioxidant present in mango leaves which is utilized for therapeutic purposes such as tachycardia arrhythmias ${ }^{[21]}$. 
Antioxidant Inhibitory Activities of Crude Ethanolic Fruit Pulp Extract of Solanum lycopersicum (Tomato) and Carica papaya (Papaya) and Mangifera indica L. (Mango) Ethanolic Leaf Extract

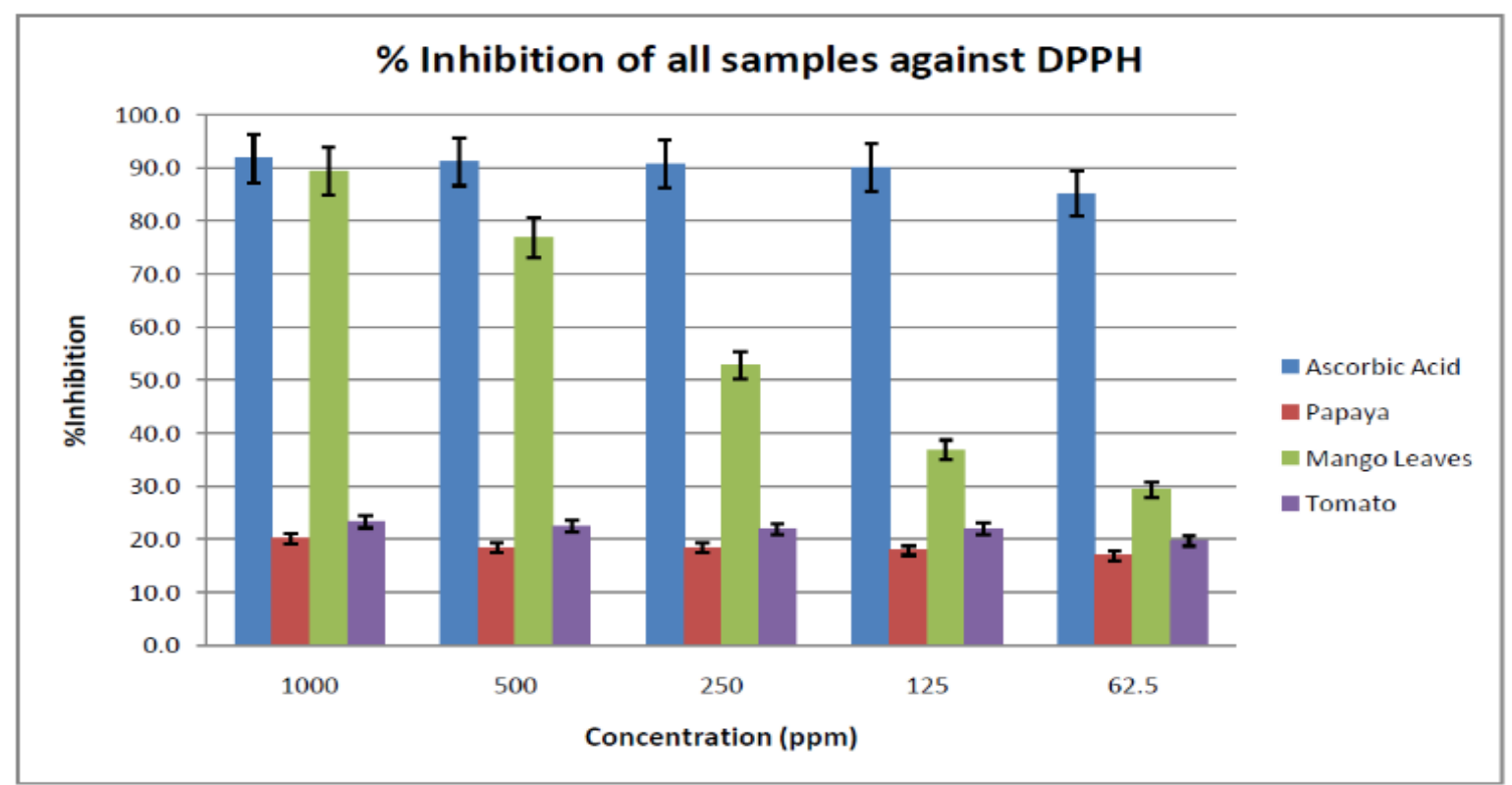

Figure 5:- Antioxidant Activity of all samples against DPPH radical. Ascorbic Acid served as the standard. Values were expressed as mean \pm standard deviation (mean \pm SD) $(n=6)$.

One Factor ANOVA result shows that there is significant difference among the selected botanical extracts and the positive control in terms of percentage of inhibition against DPPH Radical ( $\mathrm{F}=33.757 ; \mathrm{p}=0.000)$ wherein among the selected botanical extracts, the Mangifera indica L. (Mango) has the highest percent of inhibition $(\mathrm{M}=57.02)$ while the Carica papaya (Papaya) has the lowest inhibition $(\mathrm{M}=18.32)$.

In the present study conducted, selected botanical extracts were tested in terms of their antioxidant and inhibitory activity, after being tested in in vivo, for practical purposes. Since, the hearbeat/min was remarkably affected by the crude ethanolic extract of Mangifera indica L. (Mango) leaves, we checked if the antioxidant activity correlates and influence the heartbeat/min of the Daphnia magna since antioxidant agents play an important protective role in heart disease $^{[47]}$.

\begin{tabular}{|c|c|c|c|c|c|c|}
\hline & \multicolumn{5}{|c|}{ ASCORBIC ACID } & \multirow{2}{*}{ DPPH } \\
\hline Concentration(ppm) & 1000 & 500 & 250 & 125 & 62.5 & \\
\hline \multirow{6}{*}{ Absorbance } & 0.0500 & 0.0590 & 0.0540 & 0.0620 & 0.0880 & 0.617 \\
\hline & 0.0530 & 0.0520 & 0.0550 & 0.0610 & 0.0880 & 0.618 \\
\hline & 0.0490 & 0.0510 & 0.0570 & 0.0590 & 0.0930 & 0.600 \\
\hline & 0.0500 & 0.0530 & 0.0540 & 0.0590 & 0.0910 & 0.595 \\
\hline & 0.0460 & 0.0520 & 0.0570 & 0.0600 & 0.0890 & 0.606 \\
\hline & 0.0490 & 0.0540 & 0.0580 & 0.0610 & 0.0930 & 0.597 \\
\hline Average & 0.0495 & 0.0535 & 0.0558 & 0.0603 & 0.0903 & 0.606 \\
\hline SD & 0.00226 & 0.00288 & 0.00172 & 0.00121 & 0.00234 & 0.0100 \\
\hline$\%$ Inhibition & 91.8 & 91.2 & 90.8 & 90.0 & 85.1 & \\
\hline
\end{tabular}

Figure 6:- DPPH Absorbance at $540 \mathrm{~nm}$ of Ascorbic Acid

\begin{tabular}{|c|c|c|c|c|c|}
\hline & \multicolumn{5}{|c|}{ Mango Leaves } \\
\hline Concentration(ppm) & $\mathbf{1 0 0 0}$ & $\mathbf{5 0 0}$ & $\mathbf{2 5 0}$ & $\mathbf{1 2 5}$ & $\mathbf{6 2 . 5}$ \\
\hline \multirow{3}{*}{ Absorbance } & 0.0650 & 0.127 & 0.283 & 0.384 & 0.433 \\
\cline { 2 - 6 } & 0.0660 & 0.134 & 0.281 & 0.378 & 0.425 \\
\cline { 2 - 6 } & 0.0670 & 0.153 & 0.291 & 0.382 & 0.430 \\
\cline { 2 - 6 } & 0.0660 & 0.151 & 0.286 & 0.389 & 0.425 \\
\cline { 2 - 6 } & 0.0640 & 0.147 & 0.283 & 0.380 & 0.431 \\
\cline { 2 - 6 } & 0.0600 & 0.129 & 0.291 & 0.382 & 0.425 \\
\hline Average & 0.0647 & 0.140 & 0.286 & 0.383 & 0.428 \\
\hline SD & 0.00250 & 0.0115 & 0.00431 & 0.00378 & 0.00360 \\
\hline \% Inhibition & 89.3 & 76.9 & 52.8 & 36.8 & 29.3 \\
\hline
\end{tabular}


Figure 7:- DPPH absorbance at $540 \mathrm{~nm}$ of Mangifera indica L.

\begin{tabular}{|c|c|c|c|c|c|}
\hline & \multicolumn{5}{|c|}{ Tomato } \\
\hline Concentration(ppm) & $\mathbf{1 0 0 0}$ & $\mathbf{5 0 0}$ & $\mathbf{2 5 0}$ & $\mathbf{1 2 5}$ & $\mathbf{6 2 . 5}$ \\
\hline \multirow{3}{*}{ Absorbance } & 0.462 & 0.477 & 0.471 & 0.473 & 0.495 \\
\cline { 2 - 6 } & 0.474 & 0.472 & 0.475 & 0.473 & 0.465 \\
\cline { 2 - 6 } & 0.460 & 0.475 & 0.468 & 0.475 & 0.486 \\
\cline { 2 - 6 } & 0.467 & 0.478 & 0.477 & 0.479 & 0.495 \\
\cline { 2 - 6 } & 0.455 & 0.474 & 0.470 & 0.479 & 0.489 \\
\cline { 2 - 6 } & 0.470 & 0.445 & 0.477 & 0.455 & 0.486 \\
\hline Average & 0.465 & 0.470 & 0.473 & 0.472 & 0.486 \\
\hline SD & 0.00698 & 0.0125 & 0.00385 & 0.00891 & 0.0111 \\
\hline \% Inhibition & 23.3 & 22.4 & 21.9 & 22.0 & 19.7 \\
\hline
\end{tabular}

Figure 8:- DPPH Absorbance at $540 \mathrm{~nm}$ of Solanum Lycopersicum

\begin{tabular}{|c|c|c|c|c|c|}
\hline & \multicolumn{5}{|c|}{ Papaya } \\
\hline Concentration(ppm) & $\mathbf{1 0 0 0}$ & $\mathbf{5 0 0}$ & $\mathbf{2 5 0}$ & $\mathbf{1 2 5}$ & $\mathbf{6 2 . 5}$ \\
\hline \multirow{3}{*}{ Absorbance } & 0.487 & 0.487 & 0.497 & 0.499 & 0.506 \\
\cline { 2 - 6 } & 0.465 & 0.491 & 0.489 & 0.458 & 0.497 \\
\cline { 2 - 6 } & 0.491 & 0.492 & 0.501 & 0.509 & 0.509 \\
\cline { 2 - 6 } & 0.482 & 0.500 & 0.492 & 0.505 & 0.504 \\
\cline { 2 - 6 } & 0.488 & 0.498 & 0.496 & 0.504 & 0.517 \\
\cline { 2 - 6 } & 0.490 & 0.496 & 0.489 & 0.510 & 0.487 \\
\hline Average & 0.484 & 0.494 & 0.494 & 0.498 & 0.503 \\
\hline SD & 0.00975 & 0.00486 & 0.00482 & 0.0197 & 0.0103 \\
\hline \% Inhibition & 20.1 & 18.4 & 18.4 & 17.8 & 16.9 \\
\hline
\end{tabular}

Figure 9:- DPPH absorbance at $540 \mathrm{~nm}$ of Carica papaya

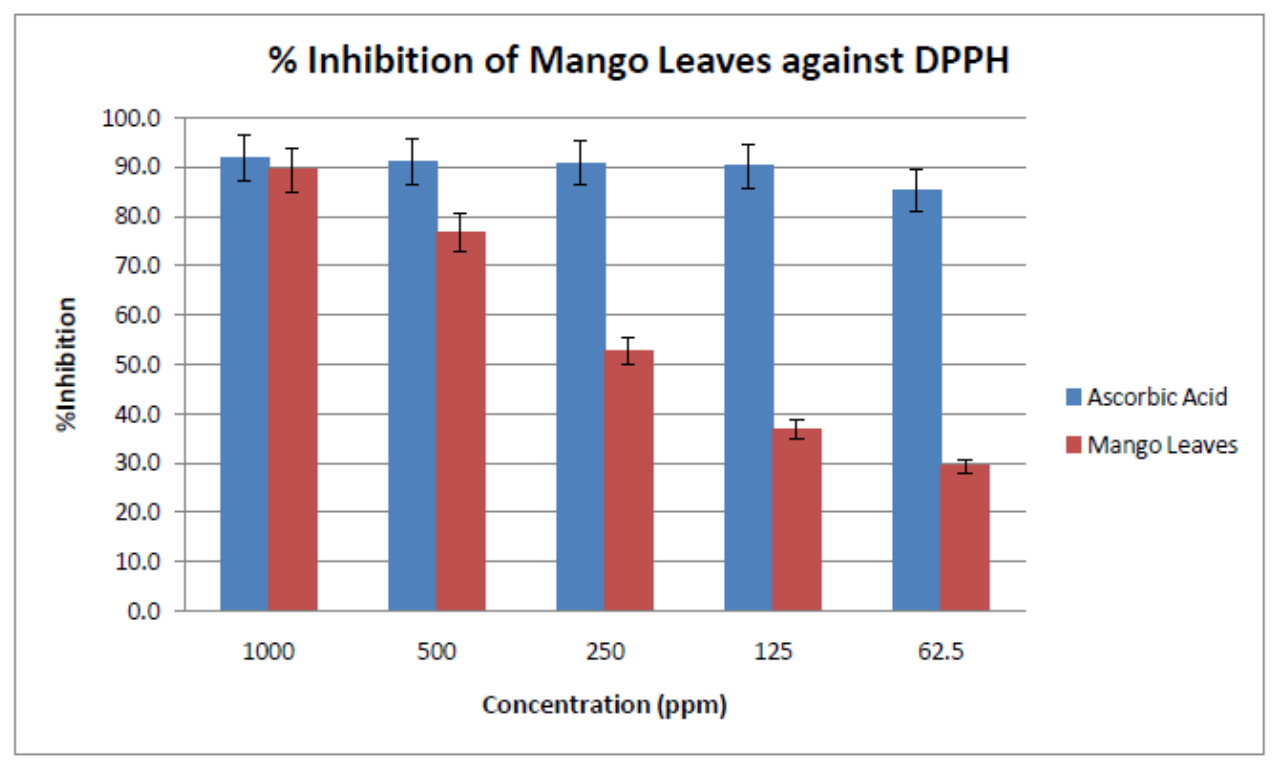


Figure 10:- Antioxidant Activity of Mangifera indica L. (Mango) against DPPH radical. Ascorbic Acid served as the standard. Values were expressed as mean \pm standard deviation (mean \pm SD) $(n=6)$.

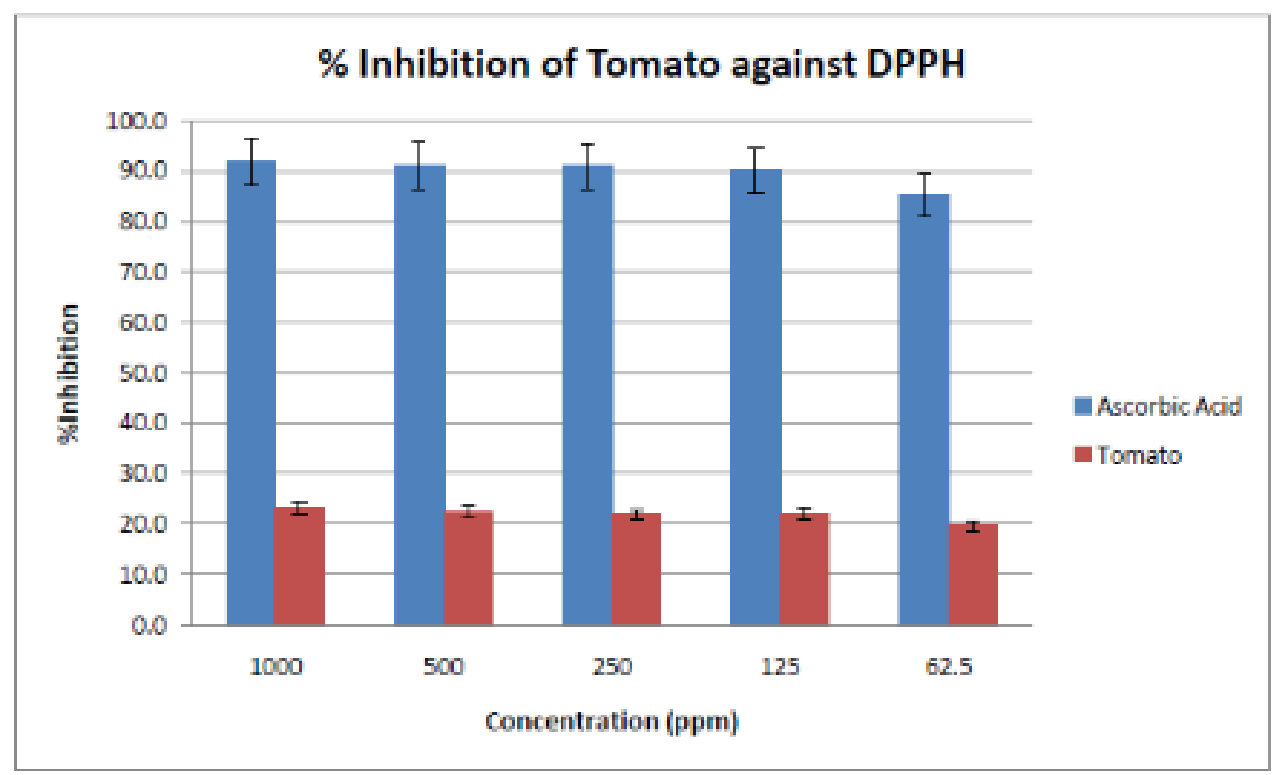

Figure 11:- Antioxidant Activity of Solanum lycopersicum (Tomato) against DPPH radical. Ascorbic acid served as the standard. Values were expressed as mean \pm standard deviation (mean \pm SD) $(n=6)$.

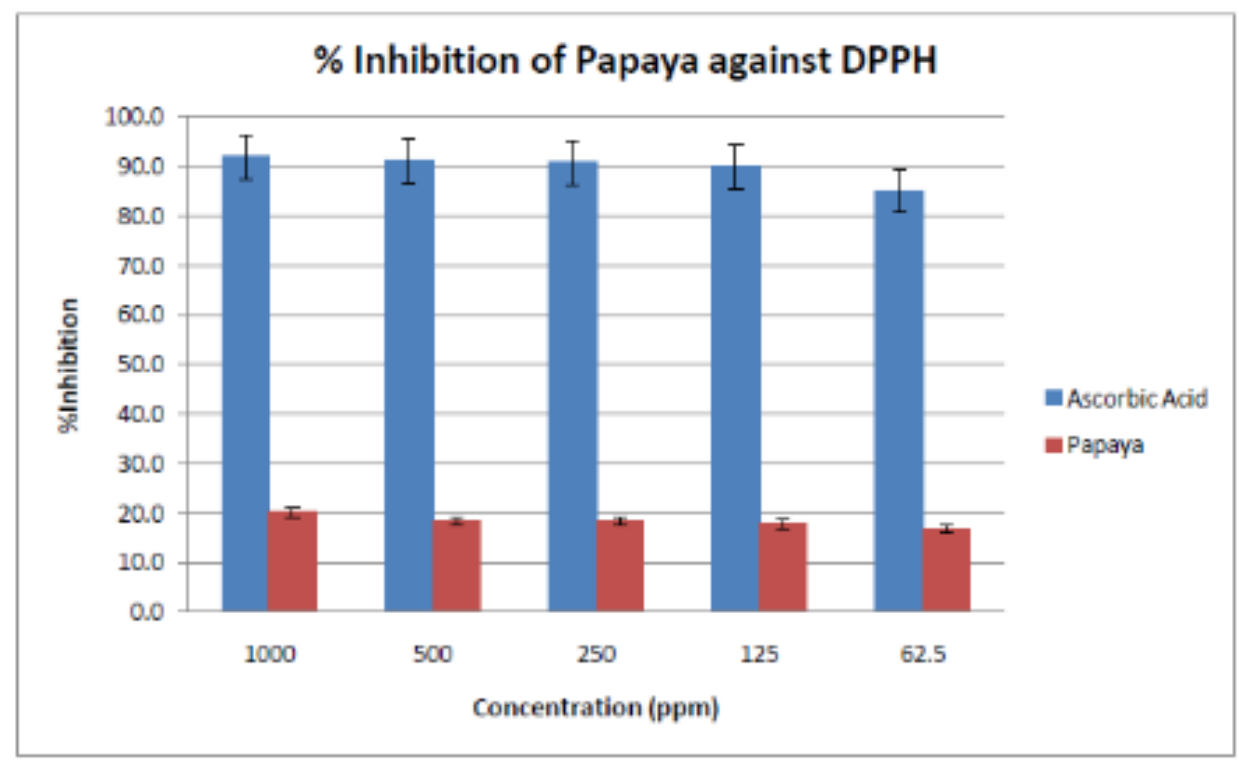

Figure 12:- Antioxidant Activity of Carica papaya (Papaya) against DPPH radical. Ascorbic Acid served as the standard. Values were expressed as mean \pm standard deviation (mean $\pm \operatorname{SD})(n=6)$.

Table 3:- \% Inhibition of the Crude Ethanolic Fruit Pulp Extract of Solanum lycopersicum (Tomato), Carica papaya (Papaya), and Mangifera indica L. (Mango) leaves

\% Inhibition of Selected Botanical Extracts against DPPH Radical

\begin{tabular}{|l|c|c|c|c|c|}
\hline Concentration(ppm) & 1000 & 500 & 250 & 125 & 62.5 \\
\hline Ascorbic Acid & 91.8 & 91.2 & 90.8 & 90 & 85.1 \\
\hline Mangifera indica L. (Mango) Leaves & 89.3 & 76.9 & 52.8 & 36.8 & 29.3 \\
\hline
\end{tabular}




\begin{tabular}{|l|c|c|c|c|c|}
\hline Solanum lycopersicum (Tomato) & 23.3 & 22.4 & 21.9 & 22 & 19.7 \\
\hline Carica papaya (Papaya) & 20.1 & 18.4 & 18.4 & 17.8 & 16.9 \\
\hline
\end{tabular}

As shown in Table 3, the three selected botanical extracts elicit remarkable antioxidant activity. Findings in the DPPH assay revealed that the crude ethanolic extract of Solanum lycopersicum (Tomato), Carica papaya (Papaya), and Mangifera indica L. (Mango) led to greater losses of DPPH absorbance at $540 \mathrm{~nm}$. The remarkable antioxidant activity or the percent of inhibition reflected by the DPPH assay was clearly observed predominantly in the crude ethanolic extract of Mangifera indica L. (Mango) leaves. On the other hand, the least losses of DPPH absorbance at $540 \mathrm{~nm}$ was found to be in the crude ethanolic fruit pulp extract of Carica papaya (Papaya), whereas, moderate losses of DPPH absorbance at $540 \mathrm{~nm}$ was found to be in the crude ethanolic fruit pulp extract of Solanum lycopersicum (Tomato).

It was observed that there was an extremely significant difference on the $\%$ of inhibition antioxidant activities among the three selected botanical extracts (Mangifera indica L., Solanum lycopersicum, and Carica papaya). Apparently, there was a minimal difference in the antioxidant activity between the crude fruit pulp ethanolic extract of Solanum lycopersicum (Tomato) and Carica papaya (papaya) as confirmed by one-way analysis of variance (ANOVA).

In this present study, it was noticed that the average heartbeat/min of Daphnia magna conforms to the findings of the antioxidant activity of each selected botanical extracts. It was revealed that the selected botanical extracts of Solanum lycopersicum (Tomato), Carica papaya (Papaya), and Mangifera indica L. (Mango) which showed remarkable antioxidant activity were in agreement with its anti-arrhythmic properties as shown in the previous figures $(2,3,4,6)$.

Based on the literature survey, antioxidants with free radical scavenging activities may have great significance in the protection and therapeutic of diseases involving free radicals ${ }^{[48]}$. Antioxidants prevent oxidative stress, caused by free radicals, which damage cells and vital biomolecules ${ }^{[49]}$. They terminate chain reactions triggered by free radicals by removing free radical intermediates and inhibit other oxidation reactions. Since, the selected botanical extracts possess potential antioxidant inhibition activity, they will minimize or prevent deleterious effects of the reactive oxygen free radicals (ROS) which exhibit influence in many cardiovascular diseases ${ }^{[49,48,50]}$. Both atrial fibrillation and heart failure, a condition with increased arrhythmic risk, are associated with excess amount of reactive oxygen species (ROS). ROSs participate in the process of damage to the sarcoplasmic membrane and increased intracellular calcium during arrhythmia, thereby causing delay and early after depolarization. While ROS seems to be able to cause arrhythmias, at least when externally supplied, and there are some possible alterations that can explain the arrhythmogenic substrate created by ROS. ROS elevation affects several ionic currents in cardiomyocytes. The effect of ROS on total and late Na+ current is important and can be arrhythmogenic. As, the ROS can downregulate cardiac sodium channels and mitochondrial antioxidants can reverse this effect ${ }^{[5]}$, therefore, antioxidant acitivity has an affirmation to the cardio rhythmic pacemaking activities of the heart.

The mechanisms of the secondary metabolites and other chemical constituents of medicinal plants account for their medicinal value ${ }^{[51]}$. These phytochemicals or secondary metabolites are bioactive compounds found in plants that work with nutrients and dietary fibre to protect against diseases. To name a few, saponins are glycosides of both triterpene and steroids having hypotensive and cardio-depressant properties ${ }^{[52]}$ while cardiac glycosides are naturally cardioactive drugs used in the treatment of congestive heart failure and cardiac arrhythmia ${ }^{[51]}$.

The anti-tachycardia arrhythmias effect of the crude ethanolic extracts of the selected botanicals extracts have an important role in pacemaker currents in cardiac nodal tissue ${ }^{[50]}$. Blocking the calcium channel results in lowering the heart rate or a negative chronotropic effect is due to slowing down the conduction of electrical activity during the plateau phase of the action potential within the heart. In addition, the anti-arrhythmic effects of calcium-channel blockers (Class IV anti-arrhythmics) are due to their ability to decrease the firing rate of aberrant pacemaker sites and are related to their ability to decrease conduction velocity and prolong repolarization within the heart, especially at the atrioventricular node. Prolonging repolarization at the atrioventricular node could help to block reentry mechanisms, which can cause supraventricular tachycardia ${ }^{[53]}$.

It can be concluded based on the results of this present study, that antioxidant activity of the crude ethanolic extracts of Solanum lycopersicum (Tomato), Carica papaya (Papaya), and Mangifera indica L. (Mango) had greatly 
influenced the cardiac rhythmic pacemaking activities of Daphnia magna induced by tachycardia arrhythmia which is a disease associated with oxidative stress which is commonly the target of antioxidant activity. Should this be the case, new possible upstream therapeutic target for treatment of arrhythmia is oxidative stress. Oxidative stress promotes myocardial fibrosis ${ }^{[54]}$. Biophysical properties of the extracellular matrix (ECM) are important factors that can affect the propagation of the action potential in the heart.

Moreover, it can also be inferred from the result of this study that crude ethanolic extract of selected botanicals (Solanum lycopersicum, Carica papaya, Mangifera indica L.) could be considered an effective agent in the prevention of various cardiovascular diseases associated with oxidative stress, including tachycardia-arrhythmia.

Table 4:- Elemental Analysis of Ethanolic Fruit Pulp Extract of Solanum lycopersicum (Tomato) and Carica papaya (Papaya) and Mangifera indica L. (Mango) Ethanolic Leaf Extract

\begin{tabular}{|l|c|c|c|}
\hline Elements & Solanum lypersicum & Carica papaya & Mangifera indica L. \\
\hline $\mathrm{Ca}$ & \multicolumn{3}{|c|}{ Relative composition } \\
\hline $\mathrm{Mg}$ & 1.07 & 0.25 & 6.47 \\
\hline $\mathrm{P}$ & 2.71 & 1.27 & 2.44 \\
\hline $\mathrm{Fe}$ & 0.58 & 0.19 & 0.20 \\
\hline
\end{tabular}

Table 4 shows the elements present in the ethanolic crude fruit pulp of Solanum lycopersicum (Tomato), Carica papaya (Papaya) and Mangifera indica L. (Mango) leaf extract. It was noted that $\mathrm{Ca}^{2+}$ and $\mathrm{Mg}^{2+}$ were found to be the highest relative composition of Mangifera indica L. (Mango). Results of this revealed that Mangifera indica L. (Mango) lowers the heartbeat of Daphnia magna. The use of $\mathrm{Mg}^{2+}$ is widely used in the prevention and/or treatment of cardiac arrhythmias. $\mathrm{Mg}^{2+}$ exerts its antiarrhythmic effect via modulation of myocardial excitability is widely accepted in cardiovascular medicine applications ${ }^{[55]}$. Oral intake of $\mathrm{Mg}^{2+}$ supplementation is inexpensive, relatively safe, and well tolerated. Moreover, the effects of calcium supplements similarly produce small beneficial effects on blood pressure ${ }^{[55,56]}$ and on circulating cholesterol fractions ${ }^{[57]}$ which might result in reduced cardiovascular events [55].

\section{Conclusion:-}

From the result of cardiotoxicity assessment of the selected botanical extracts of the fruit pulp of Solanum lycopersicum (Tomato) and Carica papaya (Papaya), and Mangifera indica L. (Mango) leaves extracts. It is clearly observed that the selected botanical extracts are safe and non-toxic to Daphnia magna. It is assumed that the antitachycardia arrhythmias effect on the heartrate/min of the test specimens Daphnia magna (water flea) may be due to the secondary metabolites found on the selected botanical extracts. Moreover, it may also be influenced by such trace elements found on the selected botanical extracts such as calcium, potassium, and magnesium content. It was revealed that antioxidant activities of the selected botanical extracts had significant influence on the heartbeat/min of the Daphnia magna since antioxidant agents play an important protective role in heart disease.

The results indicated that the selected botanicals are dose-dependent which means that the higher dosage, the greater effect to the heartbeat rates. The remarkable antioxidant activity or the percent of inhibition reflected by the DPPH assay was clearly observed predominantly in Mangifera indica L. (Mango). On the other hand, the least losses of DPPH absorbance at $540 \mathrm{~nm}$ was found to be in Solanum lycopersicum (Tomato) and Carica papaya (Papaya).

Results showed that the increase in treatment concentrations and time exposure had positive response against cardiac tachycardia arrhythmias-induced myogenic heart of Daphnia magna which behaves similarly to the heartrate of humans.

\section{Recommendations:-}

On the basis of findings and conclusions, the following recommendations are offered for consideration:

1. Quantitative determination analysis of the compounds should also be considered in determining the phytochemicals or secondary metabolites present in the selected plant extracts to explore other compounds and its effects on human health.

2. Other mechanisms of arrhythmia should be further explored so as to have new and effective therapeutic targets for treatment of all types of arrhythmia. 
3. Further investigation on other animal models and clinical trials are required to obtain drug leads.

\section{References:-}

1. U.S. Department of Health and Human Resources. (2003). Cardiovascular Risk in the Filipino Community. pp.5-10.

2. Norgren, L., Hiatt, W.R., Dormandy, J. A., Nehler, M. R., Harris, K. A., \& Fowkes, F. G. (2007). TASC II Working Group: Inter-society consensus for the management of peripheral arterial disease (TASC II). J Vasc Surg, 45, 5-67.

3. Fernández-Falgueras, A., Sarquella-Brugada, Brugada, J., Brugada, R., \& Campuzano, O. (2017). Cardiac Channelopathies and Sudden Death: Recent Clinical and Genetic Advances. Biology 2017, 6(7), 2-3.

4. Khori, V. \& Nayebpour, M. (2007). Effect of artemisia absinthium on electrophysiological properties of isolated heart of rats. Physiology \& Pharmacology Journal, 10(4), 310-311.

5. Sovari, A. A. (2016). Cellular and Molecular Mechanisms of Arrhythmia by Oxidative Stress. Cardiology Research and Practice, 3-5.

6. Canton, M., Menazza, S., Sheeran, F. L., de Laureto, P. P., Di Lisa, F., \& Pepe, S. (2011). Oxidation ofmyofibrillar proteins in human heart failure. Journal of the American College of Cardiology, 57(3), 308-309.

7. De Luna, A.B. \& Elosua, R. (2012). Sudden death. Rev. Esp. Cardiol, 65, 1039-1040). Evidence. The American Journal of Medicine, 124, 284-289.

8. Naz, A., Butt, M.S., Sultan, M.T., Qayyum, M.M.S., Rai Shahid Niaz, R.S. (2014). Watermelon Lycopene and Allied Health Claims. EXCLI Journal, 13,650-666.

9. Malviya, N. (2014). Isolation and Quantification of Lycopene from Watermelon, Tomato and Papaya. Research Journal of Recent Sciences, 3, 68-70.

10. Lilwani, S. \& Vrinda Nair, V. (2015). Extraction and Isolation of Lycopene Form Various Natural Sources. Journal of Biotechnology and Biochemistry, 1(5), 49-51.

11. Petyaev, I.M. (2015). Lycopene Deficiency in Ageing and Cardiovascular Disease. Oxidative Medicine and Cellular Longevity, 1-6.

12. SESSO, H.D. (2006). Carotenoids and cardiovascular disease: what research gaps remain? Curr Opin Lipidol, 17, 11-16.

13. Das, S. Otani, H. Maulik, N., \& Das, D.H. (2005). Lycopene, tomatoes, and coronary heart disease. Free Rad Res, 39, 449-455.

14. Riccioni, G., Mancini, E., Di Ilio, T., Bucciarelli, N., \& Orazio, N. (2008). Protective effect of lycopene in cardiovascular disease. European Review for Medical and Pharmacological Sciences, 12, 183-185.

15. Zhang, D. \& Hamauzu, Y. (2004) Phenolics, Ascorbic Acid, Carotenoids and Antioxidant Activity of Broccoli and Their Changes during Conventional and Microwave Cooking. Food Chemistry, 88, 503-509.

16. Omoni, O. \& Aluko, R. E. (2005). The anti-carcino-genic and anti-atherogenic effects of copene: A review, Trends Food Sci Tech, 16, 344-350.

17. Parvez, M. G.M. (2016). Pharmacological Activities of Mango (Mangifera Indica): A Review. Journal of Pharmacognosy and Phytochemistry, 5(3), 01-07.

18. Mirza, R. H., Chi, N., \& Chi, Y. (2013). Therapeutic Potential of the Natural Product Mangiferin in Metabolic Syndrome. Journal of Nutritional Therapeutics, 2, 74-79.

19. Daud, N.H., Aung, C.S., Hewavitharana, A.K., Wilkinson, A.S., Pierson, J.T., Roberts-Thomson, S.J. (2010). Mango extracts and the mango component mangiferin promote endothelial cell migration. J Agric Food Chem, 58(8), 5181-6.

20. Masibo, M. \& He, Q. (2008). Major mango polyphenols and their potential significance to human health. Compr Rev Food Sci Food Saf., 7, 309-319.

21. Bhatt, L., Sebastian, B., \& Joshi, V. (2017). Mangiferin protects rat myocardial tissue against cyclophosphamide induced cardiotoxicity. Journal of Ayurveda and Integrative Medicine, 8(2), 62-67.

22. Pelchovitz, D.J. \& Goldberger, J.J. (2011). Caffeine and Cardiac Arrhythmias: A Review of the Evidence. The American Journal of Medicine, 124, 284-289.

23. Kaas, B., Krishnarao, K., Marion, E., Stuckey, L., \& Kohn, R. (2009). Effects of melatonin and ethanol on the heart rate of Daphnia magna. Impulse: The Premier Journal for Undergraduate Publications in the Neurosciences, 1 .

24. Cooke, I.M. (2002). Reliable, responsive pacemaking and pattern generation with minimal cell numbers: the crustacean cardiac ganglion. Biol Bull, 202,108-36. 
25. Pirow, R., Bäumer, C., \& Paul, R.J. (2004). Crater landscape: two-dimensional oxygen gradients in the circulatory system of the microcrustacean Daphnia magna. J Exp Biol, 204, 4393-405.

26. Gard, A.L., Lenz, P.H., Shaw, J.R., \& Christie, A.E. (2009) Identification of putative peptide Ikenaka, Y., Eun, H., Ishizaka, M., Miyabara, Y. (2006). Metabolism of pyrene by aquatic crustacean, Daphnia magna. Aquat Toxicol, 80, 158-65.

27. Vaidya, A. R., Gurenlian, L., Brady, L., Romero, N. E., \& Kohn, R. (2009). Cardioactive effects of diphenhydramine and curcumin in Daphnia magna. Impulse: The Premier Journal for Undergraduate Publications in the Neurosciences, 1-5.

28. Periyanayagam, K., Karthikeyan, V., Jegadeesh, S., \& Gracelet, J.R. (2014). Cardio protective effect of the leaves of Trichosanthes cucumerina L. on Daphnia magna. International Journal of Pharmacy and Pharmaceutical Analysis, 1(2), 2.

29. Campbell, A. K. \& et. al. (2004). Lactose Causes Heart Arrhythmia in the Water Flea Daphnia Pulex. Comparative Biochemistry and Physiology, 139, 225-234.

30. Spicer, John. (2001). Development of Cardiac Function in Crustaceans: Patterns and Processes1. American Zoologist. 41. 1068-1077.

31. Kokate, C., Purohit, A.P., \& Gokhale, S.B, Pharmacognosy, Nirali Prakashan, New Delhi, 2010, 10. 28-10. 29.

32. Sofowora, A. (1993). Medicial Plants and Traditional Medicine in Africa. Spectrum Books Ltd., Ibadan, Nigeria, pp. 191-195.

33. Harborne, J.B. (1998). Phytochemical Methods. A Guide to Modern Plant Techniques of Plant Analysis. USA by Chapman and Hall. pp. 30-80

34. Periyanayagam, K. \& Karthikeyan, V. (2013). Cardio Protective Effect of the Leaves of Artocarpus heterophyllus L. on Daphnia magna. International journal of health sciences, 1(3), 1-2.

35. Krishnaiah D., Devi, T., Bono, A. \& Sarbatly, R. (2009). Studies on phytochemical constituents of six Malaysian medicinal plants. Journal of Medicinal Plants Research, 3(2), 067-072.

36. Francis, G., Kerem, Z., Makkar, H.P.S., \& Becker, K. (2012). The biological action of saponins in animal systems: A review. Br J Nutr., 88, 587-605.

37. Oyewole, O.I. \& Akingbala, P.F. (2011). Phytochemical Analysis and Hypolipidemic Properties of Jatropha tanjorensis Leaf Extract. European Journal of Medicinal Plants, 1(4), 180-185.

38. Oakenfull, D. \& Sidhu, G. (1990) Could saponins be a useful treatment for hypercholesterolemia? European Journal of Clinical Nutrition, 44, 79-88.

39. Malik, M.N.H., Alamgeer, U.S., Qayyum, A., \& Samreen, S. (2012). Phytochemical Analysis and Cardiac Depressant Activity of Aqueous Methanolic Extract of Morus nigra L. Fruit. Journal of Applied Pharmaceutical Science, 2 (12), 039-041.

40. Zhou, J.Y., Zhou, S.W., Du, X.H., Zeng, S.Y. (2012). Protective effect of total flavonoids of Seabuckthorn (Hippophaerhamnoides) in simulated high-altitude polycythemia in rats. Molecules, 17, 11585-11597.

41. Carusio, N., Wangensteen, R., Filippelli, A., Andriantsitohaina, R. (2008). Oral administration of polyphenolic compounds from cognac decreases ADP-induced platelet aggregation and reduces chronotropic effect of isoprenaline in rats. Physiol Res., 57, 517-524.

42. Saxena, M., Saxena, J., Nema, R., Singh, D., \& Gupta, A. (2013). Phytochemistry of Medicinal Plants. Journal of Pharmacognosy and Phytochemistry, 1(6), 168-175.

43. Stauth, D. (2007). Studies force new view on biology of flavonoids. Oregon State University.USA.

44. Mathai, K. (2000). Nutrition in the Adult Years. In Krause's Food, Nutrition, and Diet Therapy, 10th ed., ed. L.K. Mahan and S. Escott-Stump, 271, 274-275.

45. Subramani, S. \& Casimir, C.A.( 2002). Flavonoids and antioxidant activity of Georgia grown transcriptomics and immunohistochemistry. Gen Comp Endocrinol, 160, 271-87.

46. Okwu, D.E. (2001). Evaluation of the chemical composition of indigenous spices and flavouring agents. Global J. Pure Appl. Sci., 7(3), 455-459.

47. Dianat, M., Akbari, G., \& Badavi, M. (2013). Antidysrhthmic Effects of Gallic Acid on Cacl2-Induced Arrhythmia in Rat. International Journal of Research and Development in Pharmacy and Life Sciences, 2(6), 286-289.

48. Chidambaram, U. et al. (2013). In vitro evaluation of free radical scavenging activity of Codariocalyx motorius root extract. Asian Pacific Journal of Tropical Medicine, 188-194.

49. Agbafor, K. N. \& Nwachukwu, N. (2011). Phytochemical Analysis and Antioxidant Property of Leaf Extracts of Vitex doniana and Mucuna pruriens. Biochemistry Research International, 1-4. 
50. Akhondali, Z., Dianat, M., \& Radan, M. (2015). Negative Chronotropic and Antidysrhythmic Effects of Hydroalcoholic Extract of Lemon Balm (Melissa Officinalis L.) on CaCl2-Induced Arrhythmias in Rats. Electronic Physician, 7(1), 5.

51. Varadarajan, P \& Rathinaswamy, G \& Asirvatahm, D. (2008). Antimicrobial properties and phytochemical constituents of Rheo discolor hance. Ethnobotanical Leaflets. 12. 841-845.

52. Olaleye, M. T. (2007). Cytotoxicity and antibacterial activity of methanolic extract of Hibiscus sabdariffa. Journal of Medicinal Plants Research, 1(1), 13.

53. Eisenberg, M.J., Brox, A., \& Bestawros, A.N. (2004). Calcium Channel blockers: An update. Am J Med, $116(35)$.

54. Koli, K. Myllarniemi, M., Keski-Oja, J. \& Kinnula, V. L. (2008). Trnasforming growth factor-B activation in the lung: focus on fibrosis and reactive oxygen species. Antioxidants and redox Signalling, 10(2), 333-342.

55. Kolte, Dhaval \& Vijayaraghavan, Krishnaswami \& Khera, Sahil \& Sica, Domenic \& H. Frishman, William. (2014). Role of Magnesium in Cardiovascular Diseases. Cardiology in Review. 22. 182-192.

56. Reid I.R., Horne A., Mason B., Ames R., Bava U., Gamble G.D. Effects of calcium supplementation on body weight and blood pressure in normal older women: A randomized controlled trial. J. Clin. Endocrinol. Metab. 2005; 90:3824-3829. doi: 10.1210/jc.2004-2205.

57. Reid I.R., Mason B., Horne A., Clearwater J., Bava U., Orr-Walker B., Wu F., Evans M.C., Gamble G.D. Effects of calcium supplementation on serum lipids in normal older women-A randomized controlled trial. Am. J. Med. 2002, 112, 343-347. doi: 10.1016/S0002-9343(01)01138-X. 